\title{
The effect of inbreeding upon some growth traits in Najdi calves
}

Bahareh Taheri Dezfuli, Sima Savar Sofla, MohamadReza Mashayekhi

Agricultre and natural resources research center of Khuzestan, Ahwaz, Islamic Republic of Iran

Email: bahareh_tah2003@yahoo.com

Introduction In animals reared as small herds, accumulation of inbreeding is unavoidable. Inbreeding leads to change of genetic structure of population and to a decline in average phenotypic performance called inbreeding depression. Most evidence indicates that inbreeding adversely affects the growth and vitality of animals. Burgess et. al. (1954) showed that inbreeding had a significant effect on weaning weight of Hereford calves. Therefore, estimates of inbreeding effects are needed to adjust records of individual animals in order to increase the accuracy of the selection of breeding animals and to facilitate the analysis of data. The present study was conducted to determine the levels of inbreeding and study the effect of inbreeding on birth weight and weaning weight of Najdi calves, born in Najdi cattle Research Station, located in Khuzestan province in Iran.

Material and methods In this study, 1281 records of Najdi calves were used. Data were obtained from the Najdi cattle Research Station of Khuzestan province in Iran. Two traits were considered: birth weight (BW) and weaning weight (WW). The calves were born from 1989-2004. Fixed effects, considered in analysis were birth year, birth season, sex of calf and dam parity. The coefficient of inbreeding of the individuals and average level of population inbreeding were calculated using Pedigree software (Sargolzaei et. al., 2006) from available pedigree records of animals. The impact of inbreeding on calves birth weight and weaning weight was studied by including inbreeding as a covariate in the model.

Results Total average of birth and weaning weight of the calves were $18.08 \pm 3.17$ and $42.6 \pm 13.10 \mathrm{~kg}$, respectively. In this particular population with 1435 animals, 1244 heads were with known parents, 36 heads were with only known dams and 155 animals were with unknown parents. The average inbreeding coefficient in this population was $0.78 \%$ with the maximum level of $25 \%$. The change of the inbreeding level from 1989 to 2004 is presented in Fig. 1, shows a trend of reduction of inbreeding coefficient during studied years. In the total population, there were 186 inbred animals with the average inbreeding of $5.60 \%$, which is much more than the average inbreeding of the entire population. In addition, the average inbreeding of male and female calves were estimated of 0.75 and 1.02 , respectively. Regression coefficient of birth weight on percent of inbreeding was computed as $-0.0627 \pm 0.031 \mathrm{~kg} /$ percent of inbreeding, indicated that birth weight is depressed as 62.7 grams per percent increase in inbreeding. The same coefficient was found for weaning weight as $-0.182 \pm$ $0.12 \mathrm{~kg} / \mathrm{percent}$ of inbreeding. Ferraz et. al., (2000) reported the average inbreeding level of Santa Gertrudis herd to be 0. 0395, using 10 generations of records. Hays and Brinks (1980) reported a depressing effect of increased inbreeding on weight in beef cattle. However, in the study of Herford cattle by De Alexander and Bogart (1961), no evidence of an effect of inbreeding of the calf on birth weight.

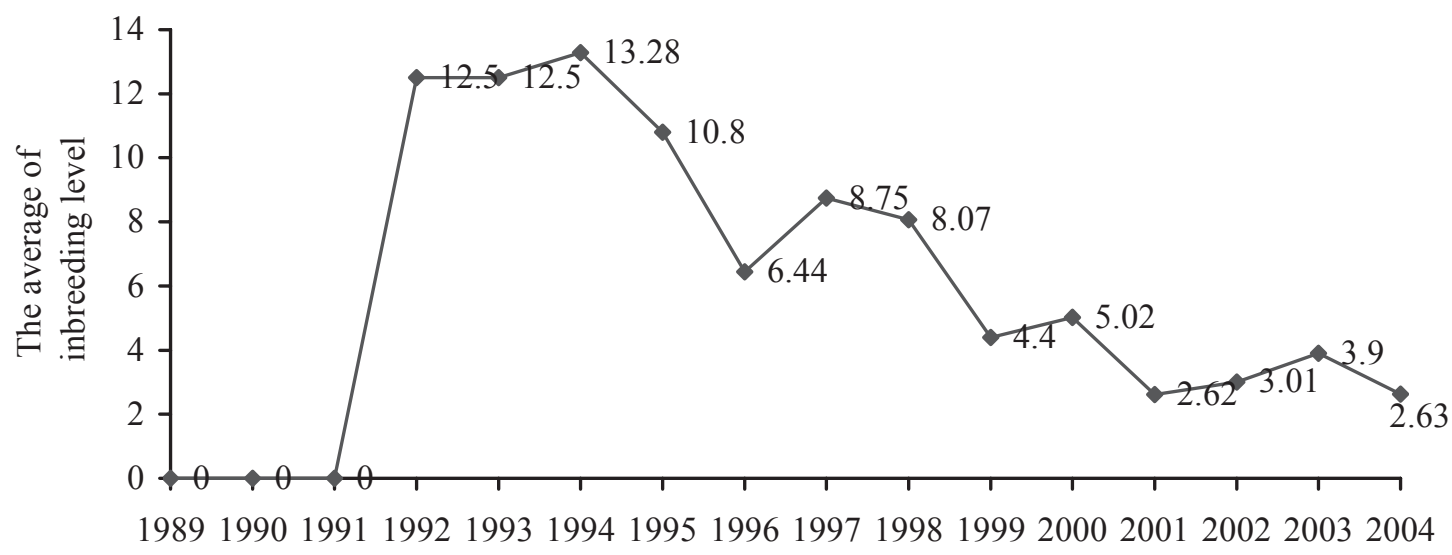

Figure 1 The change of the inbreeding level from 1989 to 2004

Conclusions The average inbreeding level of our studied population was low and the results of analysis showed that the inbreeding depression for birth weight and weaning weight was not significant. The lack of significance of the degree of inbreeding depression on studied weight traits indicate that either selection has been effective in offsetting inbreeding depression or that these traits are not subject to such inbreeding depression.

\section{References}

De Alexander, G. I. and R. Bogart. 1961. Journal of Animal Science. 20(4),702-707.

Burgess, J. B., Landblom, N. L. and H. H. Stonaker. 1954. Journal of Animal Science. 13(4),843-851.

Ferraz, J.B.S., Eler, J.P. and P.M.T. Ribeiro. 2000. Livestock Research for Rural Development. 12(2), 130-137.

Hays, W. G. and J. S. Brinks. 1980. Journal of Animal Science. 50(5),793-799.

Sargolzaei M., Iwaisaki H. and J.J. Colleau. 2006. CFC. Release 1.0. Niigata University, Niigata 950-2181, Japan. 